\title{
Synthesis and structural characterization of thin multi-walled carbon nanotubes with a partially facetted cross section by a floating reactant method
}

\author{
Y. A. Kim, T. Hayashi, M. Endo*
}

Faculty of Engineering, Shinshu University 4-17-1 Wakasato, Nagano-shi 380-8553, Japan

Y. Kaburagi

Musashi Institute of Technology,

T. Tsukada, J. Shan, K. Osato, S. Tsuruoka

Bussan Nanotech Research Institute Inc., 600 Tanaka-cho, Akishima, Tokyo, Japan

\begin{abstract}
Here we describe synthesis of very unusual multi-walled carbon nanotubes through a catalytic chemical vapor deposition method using a floating reactant method and subsequent thermal treatment up to $2600^{\circ} \mathrm{C}$ in a large quantity. Main characteristics of these nanotubes are (1) relatively wide distribution of diameters ranging from 20 to $70 \mathrm{~nm}$ and linear, long macro-morphology (aspect ratio $>100$ ), (2) highly straight and crystalline layers, (3) high purity through removal of metallic impurity, (4) very low interlayer spacing $(0.3385 \mathrm{~nm})$ and low $R$ value $\left(I_{D} / I_{G}=0.0717\right)$, (5) high $G^{\prime}$ intensity over intensity of $G$ band $\left(G^{\prime} / G=0.85\right)$ and strongly negative magnetoresistance value of $-1.08 \%$ at $77 \mathrm{~K}$ and $1 \mathrm{~T}$. The unusual microstructure of thin multi-walled carbon nanotubes with a partially cross-sectional shape caused by thermal treatment is mainly ascribed to abrupt density changes (from 1.89 to 2.1 $\mathrm{g} / \mathrm{cm}^{3}$ ) within a confined nano-sized space, accompanying with the phase separation.
\end{abstract}

Keywords: A. Carbon nanotubes, B. thermal treatment, C. Transmission electron microscopy, D. Microstructure

*Corresponding author: Tel.: +81-26-269-5201; fax: +81-26-269-5208. E-mail address: endo@endomoribu.shinshu-u.ac.jp (M. Endo) 


\section{Introduction}

Carbon nanotubes are tubular $s p^{2}$-like carbons with nanoscale dimensions that exhibit fascinating physicochemical properties that make them very promising in various applications [1-3]. The basic reason for the limited widespread application of carbon nanotubes would be their high synthetic price, basically due to the limited mass production. In this sense, a catalytic chemical vapor deposition (CCVD) method [3-22] has been extensively investigated as a powerful technique for large-scale production of single- or multi-walled carbon nanotubes (MWNTs). On top of that, this technique is more controllable and cost-efficient as compared with those of arc-discharge or laser ablation [12, 23]. Furthermore, the development of a floating reactant method, which allows a three-dimensional dispersion of hydrocarbon with the catalytic particles, enables us to produce carbon nanotubes/nanofibers in a large quantity at a relatively low cost [24-30]. The right combination of low-cost production and excellent physical properties of nanotubes make it possible to use this nanotube in various fields, such as filler in composites [31], and an anode material in lithium ion battery [32] as well as additives to electrode materials in lithium ion batteries [33] and lead-acid batteries [34]. For the purpose of widespread use of CCVD-based MWNTs for various applications, defects which deteriorate their electrical and mechanical properties and metallic impurities which are unavoidable by-products of nanotube synthesis process have to be removed. In this sense, thermal treatment has been suggested as a powerful solution for these problems [35-36].

In this study, we synthesized very unusual multi-walled carbon nanotubes with a partially facetted cross-sectional morphology using a floating reactant method and subsequent thermal treatment. A comprehensive structural characterization was carried out using a various analytical techniques.

\section{Experimental}

The synthesis of carbon nanotubes in the present study was carried out in a vertical tubular reactor, so called floating reactant method [4, 24], using a organic-metallic compound (e.g., ferrocene) as a catalyst precursor, toluene as carbon feedstock and hydrogen as carrier gas in a semi-continuous system.

A toluene solution containing organic metallic compound was fed into reactor (diameter $=$ $165 \mathrm{~mm}$ ) with a rate of $25 \mathrm{~g} / \mathrm{min}$ using a micro-feeding pump. The reaction temperature is around $1200^{\circ} \mathrm{C}$. The following thermal treatment of nanotubes was carried out at $2600^{\circ} \mathrm{C}$ for 30 minutes using a graphite-resistance furnace operating in a high-purity argon gas atmosphere in a semi-continuous system.

Field-emission SEM (FE-SEM) observations were performed using an acceleration voltage as low as $5 \mathrm{kV}$ and using a JEOL JSM-6335Fs instrument. HRTEM observations were carried out in a JEOL JEM-2010FEF instrument operated at $200 \mathrm{kV}$ (equipped with an omega 
filter, a Gatan multiscan camera and an energy dispersive X-ray - EDX - Oxford Instruments Detector). Raman spectra were recorded at room temperature under ambient conditions using a Kaiser HoloLab 5000 system; excitation source 532nm Ar-ion laser line. X-ray diffraction (JEOL-JDX3532, CuK $\alpha(\lambda=1.54056 \AA)$ ) was employed to measure the crystallinity changes.

The magnetoresistance measurements were carried out at $77 \mathrm{~K}$ at magnetic fields up to $1 \mathrm{~T}$ using a conventional four-terminal method on carbon nanotubes ( 2 and $5 \%$ )-epoxy composite ( $2 \mathrm{~mm}$ width, $10 \mathrm{~mm}$ length, $1 \mathrm{~mm}$ thickness), which is coated on polyimide film. The magnetic filed was applied along two different directions, parallel to length (longitudinal) and width (transverse) directions. The iron content of as-grown and thermally treated nanotubes was determined quantitatively by using X-ray fluorescence spectroscopy.

\section{Result and discussion}

Figure 1 shows FE-SEM images of as-grown nanotubes at different resolutions. The tubes exhibit relatively straight and long macro-morphology (aspect ratio $>100$ ) with diameters ranging from 20 to $60 \mathrm{~nm}$. A small portion of nanoparticles (see Fig. 1 (b)) and a wide distribution of tube diameters are unavoidable phenomenon for the present large-scale production of carbon nanotubes with a floating reactant method. The low-resolution TEM image shows relatively linear and constant diameter along the tube axis with a narrow hollow core $(<7 \mathrm{~nm}$ ) (Fig. 2 (a)). Main features of as-grown carbon nanotubes are their closed tip morphology (Fig. 2 (b)) and high degree of disorder (undulated fringes) (Fig. 2 (c)). The existence of metal particles in the tip of carbon nanotubes (Fig. 2 (d)) indicates that tubes are grown through a typical tip-growth mechanism $[3,12]$. In addition, rectangular morphology of metal particles suggests (Fig. 2 (d)) that round-type metal particles produced from the decomposition of organic-metallic compound are deformed into rectangular shape by high flow rate of carrier gas. Considering the high reaction temperature $\left(\mathrm{ca} .1200^{\circ} \mathrm{C}\right.$ ) and size of metal particles (below $8 \mathrm{~nm}$ ), it is reasonable to consider metal particles in a molten state. Therefore, it is possible to say that the shape of metal particles is closely related with the weight ratio of organic metallic compound, reaction temperature and flow rate.

It is noteworthy that highly disordered carbon nanotubes are made up of three microstructures (see Fig. 2 (d)): (1) Region I contain relatively straight and long fringes along tube length which is catalytically derived carbon nanotubes, (2) region II exhibits undulated fringes which is derived from deposited carbons at higher temperature of $1200^{\circ} \mathrm{C}$ (thickening process), (3) region III is highly amorphous carbon (no clear fringes) due to deposited carbon in the cooling zone of reactor. Basically, the size and the degree of distortion of the lattice fringes depend on the reaction temperature as in a typical CVD process. To study difference in microstructure of nanotubes by tube diameter (conversely speaking, the size of metal particles), two tubes with different diameters are shown in Fig. 3. Both tubes exhibit similar 
microstructures, that is, consisting of three different textures. If growth patterns of nanotubes occur as we mentioned before, it is expected the tube diameter is determined by duration time of tubes in the hottest zone of reactor, which corresponds to thickening process of nanotubes (see region II in Fig. 2 (d)) as in a typical CVD process. From the above TEM studies, as-grown nanotubes exhibit highly disordered microstructure, which would induce low physical properties, and furthermore, contains high portion of metallic impurities (ca. 1.4 wt.\%).

Through thermal treatment at $2600^{\circ} \mathrm{C}$, highly undulated fringes were structurally transformed into straight, crystalline layers for large-sized (Fig. 4 (a)) and small-sized tubes (Fig. 4 (b)). Their round tip morphology is transformed into facetted morphology (Fig. 4 (c)), accompanying with structural development. In addition, high-purity carbon nanotube is obtained through the evaporation of metallic impurity below $450 \mathrm{ppm}$. Even though these kinds of structural changes are very similar to that of thicker carbon nanofibers [38, 39], the most significant characteristics for thermally annealed nanotubes is unevenly spaced lattice fringes on one side of the hollow core, as in Figure 5 (a) and (b). In order to satisfy highly asymmetric longitudinal TEM image (Fig. 5 (a)) (Note that TEM images are two-dimensional projections), one side have a round cross-sectional shape while the others show polygonal cross-sectional shape with flat regions. Furthermore, gross defects such as bending (see short arrow in Fig. 5 (b)) and highly developed chasm-like defects along the tube length (see Fig. 5 (c)) are observed. Direct TEM observation of cross-sectional shape of annealed carbon nanotubes confirmed that the hollow core of the nanotube is not circular, rather partially facetted (Fig. 5 (d)). It is sure that chasm-like void (indicated by short arrow in Fig. 5 (d)) of cross-sectional TEM image is projected as highly developed long voids in Fig. 5 (c). The development of unusual microstructure caused by thermal treatment is ascribed to abrupt density changes ( 1.89 to $2.1 \mathrm{~g} / \mathrm{cm}^{3}$ ), which create strain and subsequently results in decreased outer diameter, expanded hollow diameter and the formation of voids. Even though as-grown state of nanotubes exhibit similar microstructures compared to that of carbon nanofiber, unusual micro-textures of thermally treated samples can be explained by limited release of strain energy due to confined space. Further study on partially facetted cross-sectional shape of carbon nanotube has to be done in order to understand the microstructure of thermally annealed carbon nanotube exactly.

The structural development of carbon nanotubes in a bulk state have been studied by X-ray diffraction and Raman spectroscopy. The (002) lines show increased intensity and move to higher angles through thermal treatment as in a typical graphitizable carbons (see Fig. 6(a)). Furthermore, increased intensity of (100) line indicates structural development of graphene 
layers while no separation of (101) and (100) lines is the lack of three-dimensional stacking. The XRD results are well consistent with Raman data (Fig. 6 (b)). Thermally treated nanotubes show an increased intensity and a decreased half width at half-maximum (HWHM) of the G band at $1582 \mathrm{~cm}^{-1}$ ( $\mathrm{E}_{\mathrm{g} 2 \mathrm{~g}}$ graphite mode), and also a decreased intensity and a decreased HWHM of the $\mathrm{D}$ band at $1350 \mathrm{~cm}^{-1}$ (the defect mode), indicating that highly disordered nanotubes are structurally developed into high crystalline nanotubes. Furthermore, the appearance of shoulder peak (D' band) at $1620 \mathrm{~cm}^{-1}$ is indirectly related with the faceting formation through the release of strain. It has to be taken into account that $\mathrm{G}^{\prime}$ band (overtone of $2 \mathrm{D}$ ) at $2700 \mathrm{~cm}^{-1}$ is very intense $\left(I_{G}, I_{G}=0.85\right)$ and also symmetric. This peak has been utilized as one of indirect indicator for the 3-dimensional (3D) stacking of graphene layers in conventional carbon materials [40] because this peak becomes asymmetric (the appearance of shoulder peak) with the development of 3D stacking as in a highly oriented pyrolytic graphite (HOPG) (see inset of Fig. 6). Based on Raman study, it is possible to conclude that thermally treated nanotubes do not show the perfect structure of graphite, for example, $A B A B$ stacking, partially due to the large curvature of small-sized nanotubes.

In order to monitor structural variation of carbon nanotubes by thermal treatment, magnetoresistance and electrical resistivity measurements were conducted because these techniques are very sensitive to a small change in the degree of structural order. For this purpose, 2 and $5 \mathrm{wt} \%$ of thermally treated tubes incorporated epoxy was placed on the polyimide film through conventional printing method. Both composites show relatively large absolute values of negative magnetoresistance, $-1.08 \%$ and $-1.0 \%$ at $77 \mathrm{~K}$ and $1 \mathrm{~T}$ as shown in Fig. 7. The negative magnetoresistance is characteristic of turbostratic structure and indicates no correlation of $3 \mathrm{D}$ stacking of the carbon layers in the thermally treated nanotubes. These absolute values are larger than that of thermally carbon nanofibers $($ diameter $=$ ca. $500 \mathrm{~nm}$ ) heat-treated at $2900^{\circ} \mathrm{C}$ (see Figure 7 (b) in ref [38]). It suggests that the two-dimensional nature of the present nanotubes is higher than that of thick nanofibers, possibly due to smaller-sized diameter of nanotubes. As expected, both samples having magnetoresistance anisotropy ratios of approximately unity confirmed that tubes are two-dimensionally aligned (note that nanotube-epoxy mixture was placed on the surface of polyimide film through conventional printing method) (see Table 1$)$. As shown in Table 1 , low values of resistivity ratio $\left(\rho_{\mathrm{RT}} / \rho_{77 \mathrm{~K}}\right)$ imply that our nanotubes present characteristic of semi-conducting materials, even though they are thermally treated at higher temperature. This experimental result strongly supports the symmetric shape of G'-band from Raman spectra even though interlayer spacing of thermally treated nanotubes is corresponding to that of pure graphite. Therefore, it is very dangerous to evaluate structural order only by using the interlayer spacing $\left(d_{002}\right)$ because no large changes are found in this $d_{002}$ regions (saturation state). 


\section{Conclusion}

Significantly unusual multi-walled carbon nanotubes were synthesized in a large quantity through a catalytic chemical vapor deposition method, especially using a floating reactant method, and subsequent thermal treatment up to $2600^{\circ} \mathrm{C}$ in a semi-continuous system. We summarized structural factors and physical parameters of these thin multi-walled carbon nanotubes in Table 2. These structural parameters are not independent, but rather are interdependent. Remind that as-grown nanotube is highly disorder (undulated fringes) in terms of microstructure, and furthermore, contain a relatively large portion of metallic impurities. Therefore, thermal treatment was applied on as-grown carbon nanotubes in order to solve their intrinsic problems. The onset temperature of oxidation was increased by ca. $40^{\circ}$, indicating that the reactive sites including defects in nanotubes were highly decreased through thermal treatment. Large density changes with improved structural order (see Table 2) might create stress in carbon nanotubes. The liberation of concentrated stress might result in the formation of a partially faceted cross-section. Therefore, preconditions for the formation of unique carbon nanotubes with high crystallinity are (1) as-grown nanotubes contain high degree of disorder, but small fringes have to align along the tube axis. Conversely speaking, nanotubes have high graphitizabiltiy. (2) Nanotube diameter has to be smaller than $70 \mathrm{~nm}$ because larger-sized tubes (or nanofibers) showed hexagonal-like cross-sectional shape (complete polygonization).

These new types of carbon nanotube with a partially faceted shape might show high thermal and electrical properties along the tube length due to their highly developed graphene layers, even though there was no sign of 3D stacking caused by small diameter. Therefore, the application of these unusual nanotubes as a functional filler in various composites will be realized due to our stabilized large-scale production in a semi-continuous system and their stabilized physicochemical properties through thermal treatment.

\section{Acknowledgements}

This work was supported by the CLUSTER of Ministry of Education, Culture, Sports, Science and Technology. 


\section{References}

[1] Dresselhaus MS, Dresselhaus G, Eklund PC. Science of fullerenes and carbon nanotubes. Academic Press:San Diego. 1996:756-864.

[2] Iijima S. Helical microtubules of graphitic carbon. Nature 1991; 354 (7):56-58.

[3] Oberlin A, Endo M, Koyama T. Filamentous growth of carbon through benzene decomposition. J Crys Grow 1976; 32:335-349.

[4] Endo M, Shikata M. Growth of vapor-grown carbon fibers using fluid ultra-fine particles of metals. Japanese Journal of Applied Physics 1985;54(5): 507-510.

[5] Masuda T, Mukai SR, Hashimoto K. The liquid pulse injection technique: A new method to obtain long vapor grown carbon fibers at high growth rate. Carbon 1994; 31(5):783-787.

[6] Mukai SR, Masuda T, Fujikata Y, Hashimoto K. The production of vapor grown carbon fibers from a mixture of benzene, toluene and xylene using the liquid pulse injection technique. Chem Eng Sci 1994;49(24):4909-4916.

[7] Baird T, Frayer JR, Grant B. Structure of fibrous carbon. Nature 1971; 233:329-330.

[8] Boehm HP. Carbon from carbon monoxide disproportion on nickel and iron catalysts: morphological studies and possible growth mechanisms. Carbon 1973; 11: 583-590.

[9] Baird T, Frayer JR, Grant B. Carbon formation on iron and nickel foils by hydrocarbon pyrolysis-reaction at 700oC. Carbon 1974; 12:591-602.

[10] Robertson SD. Carbon formation from methane pyrolysis over some transition metal surface -I. Nature and properties of the carbon formed. Carbon 1970; 8: 365-374.

[11] Tibbetts GG. Why are carbon filaments tubular?. J. Crys. Gro. 1984; 66:632-638.

[12] Baker RTK. The formation of filamentous carbon. In Walker PL Jr., editor. Chemistry and Physics of Carbon, Vol. 14, New York: Marcel Dekker, 1978: 84-165.

[13] Baker RTK, Harries PS, Terry S. Unique form of filamentous carbon. Nature 1975; 253: 37-38.

[14] Audier M, Oberlin A, Oberlin M, Coulon M, Bonnetain L. Morphology and crystalline order in catalytic carbons. Carbon 1981; 19: 217-224.

[15] Rodriguez NM, Chambers A, Baker RTK. Catalytic engineering of carbon nanostructures. Langmuir 1995; 11:3862-3866.

[16] Murayama H, Maeda T. A novel form of filamentous graphite. Nature 1990; 345: 791-793.

[17] Baker RTK, Barber MA, Harris PS, Feates FS, Waite RJ. Nucleation and growth of carbon deposits from the nickel: catalyzed decomposition of acetylene. J. Catalysis 1972; 26: 51-62.

[18] Endo M, Takeuchi K, Igarashi S, Kobori K, Shiraishi M, Kroto HW. The production and structure of pyrolytic carbon nanotubes (PCNTs). J Phys Chem Solids 1993; 54(12): 1841-1848.

[19] Dai HJ, Rinzler AG, Nikolaev P, Thesis A, Colbert DT, Smalley RE. Single-wall nanotubes 
produced by metal-catalyzed disproportionation of carbon monoxide. Chem Phys Lett 1996; 260:471-475.

[20] Andrews R, Jacques D, Rao AM, Derbyshire F, Qian D, Fan X, et al. Continuous production of aligned carbon nanotubes: a step closer to commercial realization. Chem Phys Lett 1999; 303(5-6):467-474.

[21] Terrones M, Grobert N, Olivares J, Zhang JP, Terrones H, Kordatos K, et al. Controlled production of aligned-nanotube bundles. Nature 1997; 388:52-55.

[22] Kamalakaran R, Terrones M, Seeger T, Kohler-Redlich P, Ruhle M, Kim YA, et al. Synthesis of thick and crystalline nanotube arrays by spray pyrolysis. Appl Phys Lett 2000; $77(21): 1-3$.

[23] Huang ZP, Wang DZ, Wen JG, Sennett M, Gibson H, Ren ZF. Effect of nickel, iron and cobalt on growth of aligned carbon nanotubes. Appl Phys A 2002; 74(3):387-391.

[24] Endo M. Grow Carbon Fibers in the Vapor Phase. Chem Tech 1988; 18:568-576.

[25] Tibbetts GG, Bernnardo CA, Gorkiewicz DW, Alig RL. Effect of sulfur on the production of carbon fibers in the vapor phase. Carbon 1994; 32:569-576.

[26] Collins S, Brydson R, Rand B. Structural analysis of carbon nanofibers grown by the floating reactant method. Carbon 2002; 40:1089-1100.

[27] Ci L, Wei J, Wei B, Liang J, Xu C, Wu D. Carbon nanofibers and single-walled carbon nanotubes prepared by the floating catalyst method. Carbon 2001; 39:329-335.

[28] Fan YY, Cheng HM, Wei YL, Su G, Shen ZH. The influence of preparation parameters on the mass production of vapor-grown carbon nanofibers. Carbon 2000; 38:789-795.

[29] Fan YY, Cheng HM, Wei YL, Su G, Shen ZH. Tailoring the diameters of vapor-grown carbon nanofibers. Carbon 2000; 38:921-927.

[30] Ting JM, Lake ML. Vapor-grown carbon fibers reinforced carbon composites. Carbon 1995; 33(5):663-667.

[31] Tatsumi K, Zaghib K, Abe H, Higuchi S, Ohsaki T, Sawada Y. A modification in the preparation process of a carbon whisker for the anode performance of lithium rechargeable batteries. Journal of Power Sources 1995; 54(2):425-427.

[32] Zaghib K, Tatsumi K, Abe H, Ohsaki T, Sawada Y, Higuchi S. Optimization of the dimensions of vapor-grown carbon fibers for use as negative electrodes in lithium-Ion rechargeable cells. Journal of Electrochemical Society 1998; 145(1):210-215.

[33] Endo M, Kim YA, Hayashi T, Nishimura K, Matusita T, Miyashita K, et al. Vapor-grown carbon fibers (VGCFs): Basic properties and their battery application. Carbon 2001; 39:1287-1297.

[34] Hojo E, Yamashita J, Kishimoto K, Nakashima H, Kasai Y. Improved Valve-Regulated Lead-Acid Batteries with Carbon Whisker. YUASA-JIHO Technical Review 1992; 72:23-28. 
[35] Andrews R, Jacques D, Qian D, Dickey EC. Purification and structural annealing of multiwalled carbon nanotubes at graphitization temperatures. Carbon 2001; 39(11):1681-7.

[36] Endo M, Kim YA, Fukai Y, Hayashi T, Terrones M, Terrones H, et al. Comparison study of semi-crystalline and highly crystalline multiwalled carbon nanotubes. Appl Phys Lett 2001; 79(10):1531-3.

[37] Kim YA, Hayashi T, Osawa K, Dresselhaus MS, Endo M. Annealing effect on disordered multi-wall carbon nanotubes. Chem Phys Lett 2003; 380:319-324.

[38] Speck JS, Endo M, Dresselhaus. Structure and intercalation of thin benzene derived carbon fibers. J Crys Grow 1989; 94:834-848.

[39] Endo M, Nishimura K, Kim YA, Hakamada K, Matushita T, Dresselhaus MS. Raman spectroscopic characterization of submicron vapor-grown carbon fibers and carbon nanofibers obtained by pyrolyzing hydrocarbons. J Mater Res 1999; 114 (12):4474-4477.

[40] Endo M, Kim YA, Takeda T, Hong SH, Matusita T, Hayashi T, et al. Structural characterization of carbon nanofibers obtained by hydrocarbon pyrolysis. Carbon 2001; 39:2003-10. 


\section{Figure captions}

FIG. 1. FE-SEM images of as-grown carbon nanotubes at different resolutions.

FIG. 2. As-grown carbon nanotubes: low resolution TEM images of (a) different sized nanotubes (b) closed tip morphology of nanotubes are shown; (c) high resolution TEM images exhibit a narrow hollow core along the tube axis; (d) high resolution TEM images indicate metal particle in the tip of carbon nanotubes.

FIG. 3. High resolution TEM images of (a) large-sized (ca. 60nm) and small-sized (ca. $15 \mathrm{~nm}$ ) of as-grown nanotubes

FIG. 4. Thermally annealed carbon nanotubes: high resolution TEM images of (a) large-sized and (b) small-sized nanotubes and (c) facetted tip morphology are shown. Note that highly straight graphene layers are developed along the tube length.

FIG. 5. Thermally annealed carbon nanotubes: (a)-(c) high resolution TEM images exhibit highly unevenly spaced lattice fringes on one side of the hollow core and (d) cross-sectional high resolution TEM image of nanotubes are not circular, rather show flat regions (see short arrow).

FIG. 6. (a) X-ray diffraction patterns of as-grown and thermally treated carbon nanotubes and (b) Raman spectroscopy of as-grown and thermally treated carbon nanotubes (insert one is comparative Raman lines of G' (overtone of D) from HOPG and thermally treated carbon nanotubes).

FIG. 7. Magnetoresistance for $2 \%$-and 5\%-incoporated polyimide film up to $1 \mathrm{~T}$ at $77 \mathrm{~K}$. 
Table 1 Magnetoresistance and electrical resistivity of thermally treated nanotubes-incorporated epoxy film.

\begin{tabular}{llll}
\hline I.D. & CNT2\% & CNT5\% \\
\hline$(\Delta \rho / \rho)_{\max }$ at $77 \mathrm{~K}, 1 \mathrm{~T}$ & & -1.08 & -1.00 \\
\hline Anisotropy ratio & $\mathrm{r}_{\mathrm{T}}$ & 0.96 & 0.89 \\
\cline { 2 - 4 } & $\mathrm{r}_{\mathrm{TL}}$ & 0.93 & 0.99 \\
\hline Resistivity at RT $(\Omega \mathrm{m})$ & & $0.01(0.009)$ & $0.01(0.013)$ \\
\hline Resistivity ratio $\left(\rho_{\mathrm{RT}} / \rho_{77 \mathrm{~K}}\right)$ & 0.77 & 0.76 \\
\hline
\end{tabular}

Table 2 Summarized structural and physical properties of thin multi-walled carbon nanotubes

\begin{tabular}{|c|c|c|}
\hline & Pristine nanotube & Annealed nanotube \\
\hline$d_{(002)}(\mathrm{nm})^{\mathrm{a}}$ & 0.342 & 0.3385 \\
\hline $\mathrm{L}_{\mathrm{c}}(\mathrm{nm})^{\mathrm{a}}$ & - & 13 \\
\hline Diameter $(\mathrm{nm})^{\mathrm{b}}$ & $20-70$ & $20-70$ \\
\hline Aspect ratio & $>100$ & $>100$ \\
\hline Volume density $\left(\mathrm{g} / \mathrm{cm}^{3}\right)^{\mathrm{c}}$ & 0.005 & 0.005 \\
\hline Real density $\left(\mathrm{g} / \mathrm{cm}^{3}\right)^{d}$ & 1.89 & 2.1 \\
\hline $\begin{array}{l}\text { Specific Surface area (BET) } \\
\qquad\left(\mathrm{m}^{2} / \mathrm{g}\right)^{\mathrm{e}}\end{array}$ & 28 & 28 \\
\hline $\begin{array}{l}\text { Starting temperature of } \\
\text { oxidation }^{\mathrm{f}}\end{array}$ & 560 & 600 \\
\hline Metal impurity ${ }^{\mathrm{g}}$ & 1.4 wt.\% & $<450 \mathrm{ppm}$ \\
\hline
\end{tabular}

${ }^{\mathrm{a}} d_{(002)}$ and $\mathrm{L}_{\mathrm{c}(002)}$ is interlayer spacing from XRD.

${ }^{b}$ Diameter and length of tubes were determined by FE-SEM observation.

${ }^{\mathrm{c}}$ Volume density is measured by Tapping method.

${ }^{\mathrm{d}}$ Real density is measured by a pycnometer.

${ }^{\mathrm{e}}$ Specific surface area is measured by $\mathrm{N}_{2}$ adsorption.

${ }^{\mathrm{f}}$ nanotubes are oxidized in an air atmosphere using TGA.

${ }^{\mathrm{g}} \mathrm{X}$-Ray fluorescence spectroscopy was used to determine metallic impurities. 